IDDF2020-ABS-0181 DISEASE BURDEN, RISK FACTORS, AND RECENT TRENDS OF COLORECTAL CANCER: A GLOBAL ANALYSIS OF DATA FROM 186 COUNTRIES

1Junjie Huang*, ${ }^{2}$ Harsh K Patel, ${ }^{3}$ Don Eliseo Lucero-Prisno III, ${ }^{4}$ Daniel Boakye, ${ }^{5}$ Viveksandeep Thoguluva Chandrasekar, ${ }^{6}$ Veeleah Lok, ${ }^{7}$ Xianjing Liu, ${ }^{8}$ Lin Zhang, ${ }^{9}$ Jinqiu Yuan, ${ }^{1}$ Xiang-Qian Lao, ${ }^{1}$ Shelly LA Tse, ${ }^{10}$ Wanghong Xu, ${ }^{11}$ Zhi-Jie Zheng, ${ }^{1}$ Martin CS Wong. 'Jockey Club School of Public Health and Primary Care, Faculty of Medicine, Chinese University of Hong Kong, Hong Kong; ${ }^{2}$ Department of Internal Medicine, Ochsner Clinic Foundation, USA; ${ }^{3}$ Department of Global Health and Development, London School of Hygiene and Tropical Medicine, UK; ${ }^{4}$ Department of Epidemiological Methods and Etiological Research, Leibniz Institute for Prevention Research and Epidemiology, Germany; ${ }^{5}$ Department of Gastroenterology, Mayo Clinic Arizona, USA; ${ }^{6}$ Department of Global Public Health, Karolinska Institute, Karolinska University Hospital, Sweden; 'Department of Radiology and Medical Informatics, Erasmus University Medical Centre, New Zealand; ${ }^{8}$ Melbourne School of Population and Global Health, The University of Melbourne, Australia; ${ }^{9}$ Clinical Research Centre; Scientific Research Centre, The Seventh Affiliated Hospital, Sun Yat-sen University, China; ${ }^{10}$ School of Public Health, Fudan University, China; ${ }^{11}$ Department of Global Health, School of Public Health, Peking University, China

\subsection{6/gutjnl-2020-IDDF.8}

Background This study aimed to evaluate the updated incidence, mortality, risk factors, and epidemiologic trends of colorectal cancer (CRC) for different regions and sex using publicly available data from 186 countries.

Methods The Global Cancer Observatory (GLOBOCAN) database was retrieved for the age-standardized rates (ASR) for incidence and mortality of CRC in 2018 and 2012. The prevalence of risk factors (2008-2016) was retrieved from the Global Health Observatory database. The associations between the prevalence of risk factors and incidence and mortality of CRC and were measured using beta coefficients $(\beta)$ with $95 \%$ confidence intervals (CI) generated from a univariable linear regression analysis.

Results In 2018, the highest incidence and mortality of CRC were in regions of very high human development index (HDI) (ASRs, 30.6 and 11.1) with men to women ratio of 1.4 and

\begin{tabular}{|c|c|c|c|c|c|c|c|c|c|}
\hline \multirow{2}{*}{ Outcome } & \multirow{2}{*}{ Risk factor } & \multicolumn{4}{|c|}{ Men } & \multicolumn{4}{|c|}{ Women } \\
\hline & & \multirow{2}{*}{$\frac{\beta}{7.30}$} & \multicolumn{2}{|c|}{$95 \% \mathrm{CI}$} & \multirow{2}{*}{$\frac{\boldsymbol{P}}{<0.01^{*}}$} & \multirow{2}{*}{$\frac{\beta}{4.50}$} & \multicolumn{2}{|c|}{$95 \% \mathrm{CI}$} & \multirow{2}{*}{$\frac{P}{<0.01 *}$} \\
\hline \multirow{10}{*}{$\begin{array}{l}\text { Incidence } \\
\text { (ASR) }\end{array}$} & HDI & & 6.32 & 8.28 & & & 3.94 & 5.05 & \\
\hline & GDP per capita & 4.23 & 3.30 & 5.15 & $<0.01 *$ & 2.89 & 2.39 & 3.40 & $<0.01^{*}$ \\
\hline & Alcohol consumption & 1.30 & 1.02 & 1.57 & $<0.01 *$ & 2.57 & 2.05 & 3.09 & $<0.01^{*}$ \\
\hline & Tobacco use & 0.13 & -0.10 & 0.36 & 0.27 & 0.55 & 0.42 & 0.67 & $<0.01 *$ \\
\hline & Insufficient physical activity & 0.59 & 0.34 & 0.84 & $<0.01 *$ & 0.19 & 0.07 & 0.31 & $<0.01 *$ \\
\hline & Overweight & 0.47 & 0.38 & 0.57 & $<0.01 *$ & 0.20 & 0.11 & 0.29 & $<0.01^{*}$ \\
\hline & Obesity & 1.00 & 0.77 & 1.23 & $<0.01 *$ & 0.24 & 0.11 & 0.38 & $<0.01^{*}$ \\
\hline & Diabetes & -0.51 & -1.35 & 0.33 & 0.24 & -0.57 & -0.96 & -0.18 & $<0.01^{*}$ \\
\hline & Hypertension & 0.63 & 0.09 & 1.17 & $0.02^{*}$ & -1.10 & -1.31 & -0.90 & $<0.01 *$ \\
\hline & Elevated cholesterol & 1.80 & 1.53 & 2.07 & $<0.01^{*}$ & 1.22 & 1.05 & 1.38 & $<0.01^{*}$ \\
\hline \multirow{10}{*}{$\begin{array}{l}\text { Mortality } \\
\text { (ASR) }\end{array}$} & HDI & 2.32 & 1.88 & 2.75 & $<0.01^{*}$ & 1.12 & 0.88 & 1.37 & $<0.01^{*}$ \\
\hline & GDP per capita & 1.08 & 0.68 & 1.47 & $<0.01 *$ & 0.60 & 0.39 & 0.81 & $<0.01 *$ \\
\hline & Alcohol consumption & 0.46 & 0.35 & 0.57 & $<0.01 *$ & 0.68 & 0.48 & 0.87 & $<0.01^{*}$ \\
\hline & Tobacco use & 0.11 & 0.02 & 0.19 & $0.02^{*}$ & 0.15 & 0.10 & 0.20 & $<0.01^{*}$ \\
\hline & Insufficient physical activity & 0.19 & 0.09 & 0.29 & $<0.01^{*}$ & 0.05 & 0.01 & 0.09 & $0.02^{*}$ \\
\hline & Overweight & 0.16 & 0.12 & 0.20 & $<0.01^{*}$ & 0.06 & 0.03 & 0.09 & $<0.01 *$ \\
\hline & Obesity & 0.34 & 0.25 & 0.43 & $<0.01 *$ & 0.08 & 0.04 & 0.13 & $<0.01^{*}$ \\
\hline & Diabetes & -0.11 & -0.43 & 0.21 & 0.49 & -0.08 & -0.22 & 0.05 & 0.22 \\
\hline & Hypertension & 0.45 & 0.26 & 0.65 & $<0.01 *$ & -0.23 & -0.31 & -0.15 & $<0.01^{*}$ \\
\hline & Elevated cholesterol & 0.56 & 0.44 & 0.68 & $<0.01 *$ & 0.31 & 0.24 & 0.38 & $<0.01^{*}$ \\
\hline $\begin{array}{l}\text { The analys } \\
\beta \text {, beta coe } \\
\text { with one p } \\
\text { Cl, confide } \\
\text { products. }\end{array}$ & $\begin{array}{l}\text { as conducted using univ } \\
\text { ent. The beta coefficient } \\
\text { nt increase of a certain r } \\
\text { interval; ASR, age-stan } \\
\text { than } 0.05 \text {. }\end{array}$ & & & & a tace & & & & \\
\hline
\end{tabular}

Abstract IDDF2020-ABS-0181 Figure 1 Associations between risk factors and incidence and mortality of colorectal cancer
1.2. Population in countries with higher incidence have higher alcohol consumption $(\beta=1.30,95 \%$ CI 1.02 to 1.57 for men; $\beta=0.46$, CI 0.35 to 0.57 for women), higher prevalence of tobacco use ( $\beta=0.11$, CI 0.02 to 0.19 for women), insufficient physical activity $(\beta=0.59$, CI 0.34 to $0.84 ; \beta=0.19$, CI 0.09 to 0.29$)$, overweight $(\beta=0.47$, CI 0.38 to $0.57 ; \beta=0.16$, CI 0.12 to 0.20$)$, obesity $(\beta=0.24$, CI 0.11 to $0.38 ; \beta=0.34$, CI 0.25 to 0.43$)$, and elevated cholesterol $(\beta=1.80$, CI 1.53 to $2.07 ; \beta=1.22$, CI 1.05 to 1.38 ). Similar associations were also found for mortality (figure 1). From 2012 to 2018, there was an overall increase in the trend of incidence and mortality, particularly in Asia $(+29.7 \%$ and $+17.4 \%)$ and Africa $(+24.3 \%$ and $+17.6 \%)$, and among men $(+14.6 \%$ and $+8.0 \%)$ compared with women $(+14.0 \%$ and $+4.3 \%)$.

Conclusions The variation in disease burden of CRC was associated with HDI and the prevalence of risk factors. There was an increasing trend in the incidence and mortality of CRC, particularly in regions with low and middle incomes and among men. More intensive lifestyle modifications and population-based screening are recommended for these populations.

\section{IDDF2020-ABS-0183 PROFILE TRIAL: PREDICTING OUTCOMES FOR CROHN'S DISEASE USING A MOLECULAR BIOMARKER}

${ }^{1}$ Nurulamin Noor*, 'Biljana Brezina, ${ }^{1} J u a n$ De La Revilla Negro, ${ }^{2}$ Francis Dowling, ${ }^{2}$ Leisha O'Brien, ${ }^{2}$ Sanjana Choudhury, ${ }^{2}$ Simon Bond, ${ }^{2}$ Lynne Whitehead, ${ }^{3}$ Sara Upponi, ${ }^{4}$ Kamal Patel, ${ }^{4}$ Abigail Seward, ${ }^{5}$ Christopher Probert, ${ }^{6}$ Klaartje Kok, ${ }^{7}$ John Gordon, ${ }^{8}$ Paul Lyons, ${ }^{8}$ Eoin McKinney, ${ }^{8}$ Kenneth Smith, ${ }^{8}$ James Lee, ${ }^{1}$ Miles Parkes, ${ }^{9}$ PROFlLETrial Investigators. ${ }^{1}$ Department of Gastroenterology, Cambridge University Hospitals NHS Trust, UK; ${ }^{2}$ Cambridge Clinical Trials Unit, Cambridge University Hospitals NHS Trust, UK; ${ }^{3}$ Department of Radiology, Cambridge University Hospitals NHS Trust, UK; ${ }^{4}$ Department of Gastroenterology, St George's Hospital, UK; ${ }^{5}$ Department of Gastroenterology, Liverpool University Hospitals NHS Foundation Trust, UK; ${ }^{6}$ Department of Gastroenterology, Barts and the Royal London Hospital, UK; ${ }^{7}$ Department of Gastroenterology, Royal Hampshire County Hospital, UK; ${ }^{8}$ Department of Medicine, University of Cambridge School of Clinical Medicine, UK; ${ }^{9}$ National Health Service, UK

\subsection{6/gutjnl-2020-IDDF.9}

Background The course of IBD varies substantially between individuals, but there are a lack of reliable prognostic markers to guide clinical practice. Previously, we have described a transcriptional signature detectable within peripheral blood CD8 T-cells at diagnosis, identifying two subgroups of patients, correlating with subsequent disease course. We have sought to develop a whole-blood biomarker that could re-capitulate the prognostic CD8 subgroups and then assess whether this biomarker can improve clinical outcomes by appropriately matching therapy to disease course.

Methods From a training cohort of 69 newly-diagnosed IBD patients, we simultaneously obtained a whole-blood PAXgene RNA tube and peripheral blood CD8 T-cell sample. Gene expression in both samples was measured by microarray. Statistical modelling was used to identify a transcriptional classifier in whole-blood gene expression data re-capitulating the CD8 findings and subsequently optimised into a multi-gene qPCR assay with independent validation in a second, independent cohort of 123 newly-diagnosed adult patients.

The PROFILE trial has incorporated this classifier to compare the relative efficacy of 'top-down' and 'accelerated stepup' therapy between biomarker-defined subgroups of 400 patients with newly-diagnosed Crohn's disease. 
Results Following application of statistical (machine) learning methods described, a 17 gene qPCR assay was developed and optimised. The validation cohort of 123 patients, could be classified into two distinct subgroups, IBD ${ }^{\text {hi }}$ (high risk) and $\mathrm{IBD}^{\mathrm{lo}}$ (lower risk). Irrespective of the underlying diagnosis, IBD $^{\text {hi }}$ patients experienced significantly more aggressive disease than $\mathrm{IBD}^{\mathrm{lo}}$ patients, with an earlier need for treatment escalation (hazard ratio=2.65 (CD), $3.12(\mathrm{UC})$ ).

Subsequently, this biomarker is being used to stratify therapy in the PROFILE trial, where 45 sites have been opened and at the time of writing, over 260 participants randomised - with recruitment ongoing.

Conclusions We have developed, optimised and validated a whole-blood qPCR classifier that is able to predict disease course from diagnosis in patients with IBD. This classifier is currently being used in the PROFILE trial, the first biomarker-stratified trial in Gastroenterology, and if the clinical utility of a stratified treatment approach is demonstrated this would represent a major step towards personalised therapy in IBD.

\section{IDDF2020-ABS-0204 FECAL MICROBIOTA AS A NON-INVASIVE IDENTIFICATION FOR POSTOPERATIVE ENDOSCOPIC RECURRENCE IN CROHN'S DISEASE PATIENTS}

${ }^{1}$ Haichao Wang*, ${ }^{2}$ Guorong Yan, ${ }^{1}$ Yaling Wu, ${ }^{3}$ Chen Ye, 'Deji Zhuoma, ${ }^{1}$ Pengyu Yang, ${ }^{1}$ Lei Zhang, ${ }^{4}$ Chungiu Chen, 'Xiaolei Wang. ${ }^{1}$ Department of Gastroenterology, Shanghai Tenth People's Hospital, Tongji University School of Medicine, China; ${ }^{2}$ Institute of Photomedicine, Shanghai Skin Disease Hospital, Tongji University School of Medicine, China; ${ }^{3}$ Medical College of Soochow University, China; ${ }^{4}$ Center for Difficult and Complicated Abdominal Surgery, Shanghai Tenth People's Hospital, Tongji University School of Medicine, China

\subsection{6/gutjnl-2020-IDDF.10}

Background Postoperative recurrence (POR) is extremely frequent in Crohn's disease (CD) after ileocolonic resection and usually assessed by endoscopy. However, the use of endoscopies is limited by its invasiveness. Dysbiosis of gut microbiota has been implicated in triggering POR. Here, we aimed to establish a non-invasive tool based on fecal microbiota to identify POR in Chinese CD patients.

Methods Fecal samples from 52 CD patients within 12 months after ileocecal resection were collected for gut microbiota analysis using 16S rRNA genes sequencing technology. POR was defined by a Rutgeerts score $\geq \mathrm{i} 2$ under endoscopy at the time of sample collection. Microbiota abundance at the genus level was employed to construct the model to estimate POR by logistic regression model (LRM). Leave-one-out cross validation was used to evaluate the robustness of the model. Receiver operating characteristic curve analysis was performed to test the sensitivity and specificity of the microbial model.

Results Significant reduction of microbial diversity was noted in the POR group $(p<0.05)$. In patients developing POR (27 cases), an increase of Flavonifactor.spp belonging to Ruminococcaceae family, and a reduction of 14 genera, including three short-chain fatty acid (SCFA)-producing genera belonging to Lachnospiraceae family (Stomatobaculum. spp, Oribacterium.spp and Eubacterium.spp), was observed (all $p<0.05$, (figure 1A)). Lower percentage of infliximab treatment was found in POR patients $(33.3 \%$ vs. $61.5 \%$, $p$
A

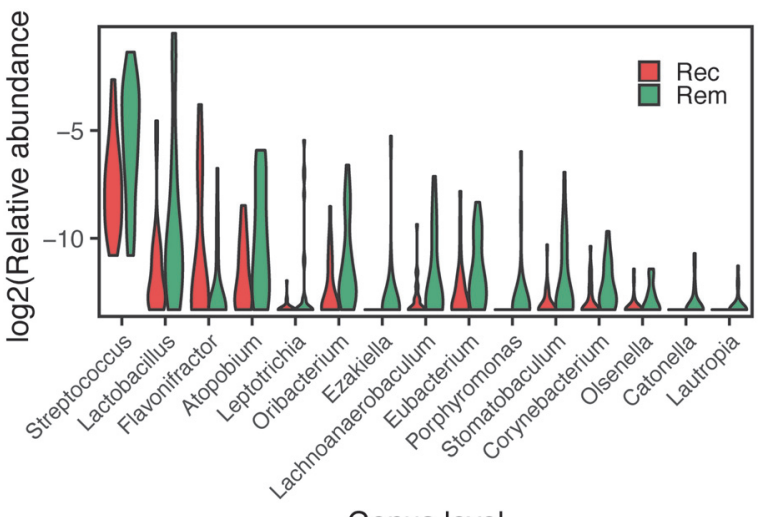

Genus level

Abstract IDDF2020-ABS-0204 Figure 1A Identification of discriminatory gut microbiota for $C D$ patients with postoperative recurrence

\section{B}

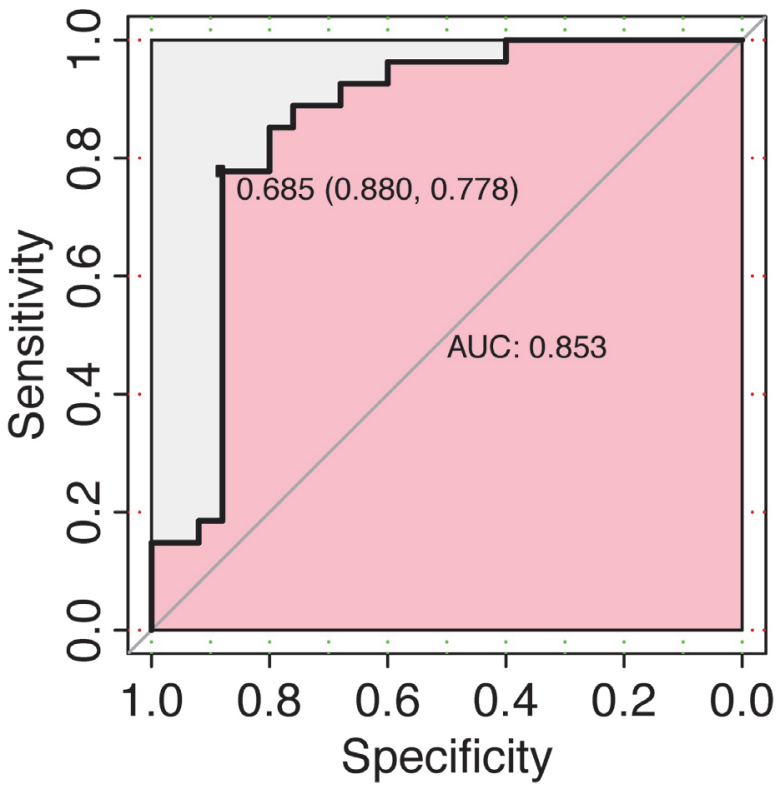

Abstract IDDF2020-ABS-0204 Figure 1B Identification of discriminatory gut microbiota for $C D$ patients with postoperative recurrence

$=0.040$ ), and closely associated with elevated abundance of Stomatobaculum.spp and Oribacterium.spp $(p<0.01)$. Both the abundance of Stomatobaculum.spp and Oribacterium.spp were negatively correlated with the counts of platelets or lymphocytes $(p<0.05)$. A score combined with discriminatory microbial species was calculated to predict POR, and achieved an area under curve value of 0.853 (0.880 for specificity, 0.778 for sensitivity, (Figure 1B)). The correlation coefficient between the observed and the predicted values was $0.651(p<0.01)$.

Conclusions POR is tightly linked to the changes in microbiota composition and diversity. Fecal microbiota, including Flavonifactor.spp and three genera of SCFA-producing Lachnospiraceae family, represents a non-invasive biomarker for diagnosis of POR, helping to prevent POR in CD patients. 